\title{
The STACC 1998 Campaign on Praesepe: The Spectroscopy
}

\author{
Thomas H. Dall \\ Institute of Physics and Astronomy, Aarhus University, Denmark
}

\begin{abstract}
The STACC campaign of 1998 on BN Cnc was an extensive international photometric campaign aimed at obtaining accurate frequencies of the pulsations in this star. To facilitate identification of the modes, parallel spectroscopic observations were made from six observatories around the world. I here present preliminary results from the spectroscopy made from the Nordic Optical Telescope. The accurate frequencies from the photometric campaign are used to obtain accurate amplitudes of the equivalent width variations. These results are to be used in an attempt to identify the pulsation modes in this star.
\end{abstract}

\section{The Equivalent Width Method}

Measuring stellar pulsations by equivalent width (EW) measurements was proposed by Bedding et al. (1996) and has been used with success by Viskum et al. (1998) on the $\delta$ Scuti star FG Virginis. The strength of stellar absorption lines are temperature dependent and the oscillations, which produce temperature variations, can thus be measured via the $\mathrm{EW}$. In the case of $\mathrm{BN}$ Cnc we have concentrated on the $\mathrm{H} \alpha$ line, because it is a very strong line known to be sensitive to the temperature oscillations, as proven in the aforementioned case of FG Vir. One of the goals of this campaign, was the building of a secure and standardized procedure for reducing and analyzing data from various different sites. For the Balmer lines, this has already been accomplished. In addition to the strong Balmer lines, a lot of weaker lines are present, including lines of sodium and iron. In the case of FG Vir these lines gave valuable additional information for the mode identification. An attempt will be made, at a later stage, to formalize the treatment of the weaker spectral lines.

\section{Frequency Analysis}

The data from NOT yields an amplitude spectrum of oscillations in $\mathrm{H} \alpha \mathrm{EW}$, shown in Fig. 1. This is expected to improve once the data from all sites have been combined. It is already evident that a lot of modes are present between 250 $\mu \mathrm{Hz}$ and $350 \mu \mathrm{Hz}$, where all the photometric modes have been found (Frandsen \& Pigulski 2000).

It is our goal to identify as many modes as possible in $\mathrm{BN}$ Cnc by comparing amplitudes in photometry and $\mathrm{H} \alpha \mathrm{EW}$. The principles of this approach have been described in Viskum et al. (1998). 


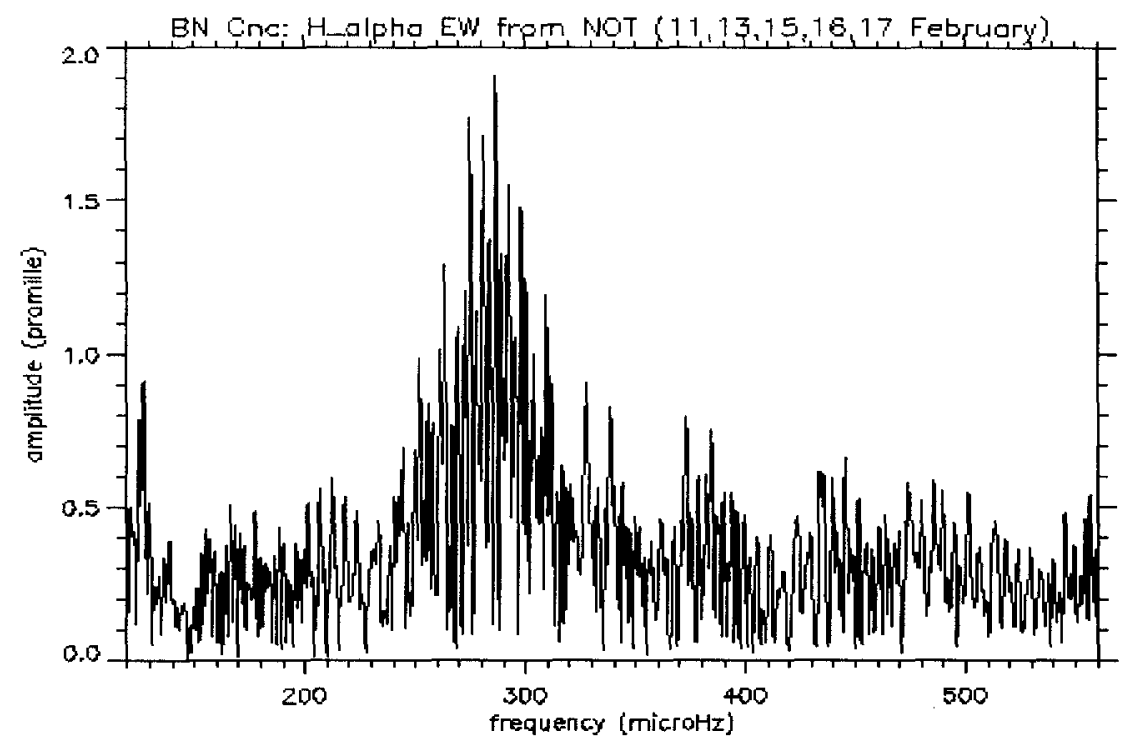

Figure 1. Amplitude spectrum of $\mathrm{H} \alpha \mathrm{EW}$ from NOT. The window is not very good, but will improve when all sites are combined.

\section{Participating Sites for the Spectroscopy of BN Cancri}

The sites and investigators participating in the spectroscopic campaign on BN Cnc are:

1) Okayama Astrophysical Observatory, Japan (Kambe, Kawanomoto, Watanabe, Fukata);

2) Vainu Bappu Observatory, India (Anupama);

3) Thüringer Landessternwarte Tautenburg, Germany (Lehmann);

4) Roque de los Muchachos Observatory, Spain (Dall);

5) McDonald Observatory, USA (Handler, Horner);

6) Dominion Astronomical Observatory, Canada (Grundah1).

The data are reduced by the observers, while the combination of data and the final analysis are to be carried out by me.

\section{References}

Bedding, T. R., Kjeldsen, H., Reetz, J., \& Barbuy, B. 1996, MNRAS, 280, 1155 Frandsen, S. \& Pigulski, A. 2000, in these proceedings, p. 473

Viskum, M., Kjeldsen, H., Bedding, T. R., Dall, T. H., Baldry, I. K., Bruntt, H., \& Frandsen, S. 1998, A\&A, 335, 549 